\title{
CLUSTERIZAÇÃO POR MÍNIMA DISTÂNCIA: UMA ABORDAGEM UTILIZANDO ALGORITMO GENÉTICO
}

\author{
Roza Maria Zoellner Lopes (GTAO / UFPR) roza.lopes@ufpr.br \\ Cassius Tadeu Scarpin (GTAO / UFPR)) cassiusts@ufpr.br \\ José Eduardo Pécora Júnior (GTAO / UFPR) pecora@ufpr.br \\ Tarek Nasser Sati (GTAO / UFPR) tarek.sati@ufpr.br
}

\begin{abstract}
Resumo
O problema de clusterização consiste no agrupamento de itens baseado em alguma característica em comum. No presente estudo realizou-se o agrupamento em clusters de pontos gerados aleatoriamente com o objetivo de obter a mínima distância entre eles. Utilizou-se a metaheurística Algoritmo Genético (AG) com soluções iniciais obtidas por heurísticas construtivas e pela metodologia $K$-means, sendo esta última melhorada pelo algoritmo Local Search. A fim de comprovar a eficiência da abordagem proposta comparouse os dados obtidos com os resultados da solução exata do problema. Os resultados mostram que o Algoritmo Genético obtém soluções para instâncias onde não é possível obter o resultado exato.
\end{abstract}

Palavras-Chaves: Clusterização, mínima distância, Algoritmo Genético.

\section{Introdução}

A clusterização é utilizada para o agrupamento de dados com características semelhantes. Possui diversas aplicações: subdivisão de problemas grandes, onde resolvê-los por completo seria inviável; a determinação da abertura de centros de distribuição com o intuito de minimizar as distâncias percorridas; agrupamento de itens por categoria, entre outros (FUENTES; CADARSO; MARÍN, 2019; GEREMEW et al., 2018).

Com a facilidade de acesso à internet os clientes estão consumindo cada vez mais produtos diversificados e exigindo rápida entrega (WANG et al., 2020). Portanto, a clusterização é importante na área de transporte, pois agrupa itens visando minimizar a distância percorrida, os custos envolvidos e o tempo de entrega.

Segundo Talbi (2009) a clusterização é um problema considerado de difícil resolução. Portanto, para casos com grandes números de pontos pode ser inviável a sua solução exata, fazendo-se necessário o uso de outras metodologias como heurísticas e metaheurísticas.

Diante disso, o presente estudo tem o objetivo de resolver o problema de clusterização por distância utilizando a metaheurística Algoritmo Genético. Para testar a eficiência do método 
os resultados obtidos foram comparados com a resolução do modelo exato resolvido com o Gurobi 8.0.

Com os resultados é possível constatar que a o Algoritmo Genético obtém a solução ótima para pequenas instâncias e consegue resolver problemas de maior escala em um tempo computacional baixo.

$\mathrm{O}$ artigo está estruturado em quatro seções. A seção 2 apresenta a revisão de literatura sobre a clusterização e o Algoritmo Genético. Na seção 3 são apresentados o problema e a metodologia proposta. A seção 4 contém a análise dos resultados obtidos. E por fim, na seção 5 apresentam-se as considerações finais do estudo.

\section{Clusterização}

Os algoritmos de cluster podem ser classificados em hierárquicos e de particionamento. $\mathrm{O}$ hierárquico obtém uma rede com uma série de divisões e o de particionamento agrupa os dados simultaneamente para otimizar de acordo com um critério ou função objetivo (NASCIMENTO; TOLEDO; CARVALHO, 2010). Os algoritmos de hierarquização mais utilizados são o link único e o link completo e o de particionamento mais utilizado é o $k$ means (JAIN, 2010).

A maioria dos algoritmos de clusterização são convertidos em problemas de otimização combinatória com o intuito de agrupar dados de acordo com uma função objetivo, porém para problemas de grande porte é difícil de se resolver computacionalmente devido à sua complexidade, portanto é necessário buscar meios de solução que demandem menor esforço computacional (JAIN, 2010).

São diversas as aplicações da clusterização na literatura, o Problema de Roteamento de Veículo Verde (GVRP) foi resolvido por Tiwari e Change (2015) utilizando uma abordagem de cluster para cada cidade do problema. Foi aplicada a recombinação de blocos com o intuito de diminuir a emissão de dióxido de carbono.

Defryn et al. (2016) resolveram o problema de roteamento colaborativo com três clientes, foi utilizada a clusterização para verificar o impacto da proximidade de clientes que pertencem a cada parceiro.

Geremew et al. (2018) solucionaram o problema de determinação de centros de distribuição para os clientes e também um centro principal pra abastecer os demais, sendo definido como 
um problema de cluster hierárquico em dois níveis. Foi proposto dois algoritmos implementáveis baseados em DCA para resolver o problema.

Gatica et al. (2018) utilizaram K-means para determinar o centro do cluster para abastecimento de veículos elétricos visando encontrar o melhor local de abertura, minimizando os custos.

Fuentes et al. (2019) usaram a clusterização para o problema de agendamento. O método consiste em primeiramente realizar uma decomposição matemática ad-hoc com base na clusterização de tempo-pessoal. Após, foi utilizado o Fix and Relax para resolver o problema resultante.

Chao, Zhihui e Baozhen (2019) resolveram o problema de roteamento de localização e inventário com janela de tempo em uma rede de distribuição de alimentos em duas etapas, a primeira para o problema de localização e a segunda para o problema de transporte. Utilizaram uma abordagem de cluster baseado em distância para aumentar a eficiência da metodologia proposta, melhorando a velocidade de convergência da solução.

Cao et al. (2020) utilizaram um algoritmo de cluster baseado na metodologia hierárquica para agrupar regiões funcionais da trajetória do eletrodo na cirurgia de estimulação cerebral profunda.

Algumas das metaheurísticas mais utilizadas para a clusterização de dados são: Tabu Search, Simulated Annealing, Greedy Randomized Adaptive Search Proce- dure (GRASP) e o Algoritmo Genético (AG) (Nascimento et al., 2010).

\subsection{Algoritmo Genético}

Os Algoritmos Evolutivos (EA) obtém soluções aproximadas em um tempo computacional baixo se comparado com os métodos exatos e conseguem ótimos resultados para problemas grandes e complexos. Um dos algoritmos EA mais utilizados para problemas de otimização é o Algoritmo Genético (Cao et al., 2020).

Em um estudo realizado por Coello em 2017 que considerou os anos de 1988 à 2017 foram encontrados 4987 artigos que utilizaram algoritmos EA para resolver problemas de otimização multiobjetivo, sendo que em $56 \%$ dos artigos foi utilizado o Algoritmo Genético. 
O AG foi proposto inicialmente por Holland em 1975 e encontra soluções com ótima qualidade para problemas NP difíceis realizando uma busca global, podendo assim, escapar da solução local (Wu et al., 2019).

O Algoritmo Genético é uma metaheurística baseada na teoria de Charles Darwin onde os seres mais aptos sobrevivem (CASTRO, 2001). A partir de soluções iniciais é realizada uma seleção aleatória de indivíduos para cruzar seus cromossomos e obter novos indivíduos. A nova população sofre alguma mutação com o intuito de diversificar as soluções encontradas (TALBI, 2009). Para obter a nova geração de indivíduos existem várias técnicas, entre elas, inserir o novo indivíduo na população e descartar o de pior função objetivo ( TALBI, 2009; BARBOZA, 2010)

Segundo Lopes, Rodrigues e Arns (2013) os passos para a implementação do AG são: Obtenção da população inicial; Seleção dos indivíduos para reprodução; Realização do cruzamento entre os indivíduos pais; Mutação da nova população e verificação dos critérios de parada.

\section{Descrição do problema}

O problema do presente artigo consiste em determinar em qual cluster cada ponto será alocado com o objetivo de obter a soma mínima das distâncias entre os pontos de cada cluster. Cada ponto pode pertencer a apenas um cluster e a distância utilizada é a euclidiana.

O problema será resolvido utilizando o Algoritmo Genético. Para testar a eficiência do método proposto foi realizada a comparação dos resultados obtidos com as soluções do modelo exato.

\subsection{Modelo matemático}

O modelo exato utilizado neste artigo foi formulado por Nascimento, Todelo e Carvalho (2010) que modificaram o modelo proposto por RAO (1971):

Tabela 1 - Índices do modelo

\begin{tabular}{l|l}
\hline \multicolumn{1}{c}{ Índices } \\
\hline$i$ & nó de partida \\
$j$ & nó de chegada \\
$k$ & cluster \\
\hline
\end{tabular}




\begin{tabular}{c|l}
\multicolumn{2}{c}{ Parâmetros } \\
\hline$n$ & número total de vértices \\
$m$ & número total de clusters \\
$d_{i_{i} j}$ & distância entre os vértices i e j \\
\hline
\end{tabular}

As variáveis de decisão são definidas como:

$x_{i, k}=\left\{\begin{array}{l}1 \text { se o vértice i pertence ao cluster } k \\ 0, \text { caso contrário. }\end{array}\right.$

$y_{i, j}=\left\{\begin{array}{l}1 \text { se os vértices } i \text { e } j \text { pertencem ao mesmo cluster. } \\ 0, \text { caso contrário. }\end{array}\right.$

Função objetivo:

$\operatorname{Min} \sum_{i=1}^{N-1} \sum_{j=i+1}^{N} d_{i j} y_{i j}$

Sujeito a

$\sum_{\mathrm{k}=1}^{\mathrm{M}} \mathrm{x}_{\mathrm{ik}}=1, \quad i=1, \ldots, N$

$\sum_{i=1}^{N} x_{i k} \geq 1, \quad k=1, \ldots, M$

$x_{i k} \in\{0,1\}, \quad i=1, \ldots, N \quad k=1, \ldots, M$

$y_{i j} \geq x_{i k}+x_{j k}-1, \quad i=1, \ldots N \quad j=i+1, \ldots, N \quad k=i, \ldots, M$

$y_{i j} \geq 0, \quad i=1, \ldots, N \quad j=i+1, \ldots, N$ 
A função objetivo visa obter a mínima distância entre os vértices do mesmo cluster. A restrição (1) determina que um vértice pode pertencer a apenas um cluster. A restrição (2) garante que o cluster $k$ terá pelo menos um vértice. As restrições (3) e (5) determinam que as variáveis $x_{i k}$ e $y_{i j}$ são binárias. A restrição (4) garante que $y_{i j}$ será 1 se tanto $x_{i k}$ quanto $x_{j k}$ forem 1 .

\section{Metodologia proposta}

A figura 1 apresenta o método proposto para a resolução do problema de clusterização. São obtidas soluções iniciais a partir de heurísticas construtivas e do k-means que tem sua solução melhorada por meio de uma busca em sua vizinhança utilizando o Local Search. Essas soluções são utilizadas para alimentar o Algoritmo Genético que realiza uma busca global para obter a solução final.

Figura 1 - Metodologia

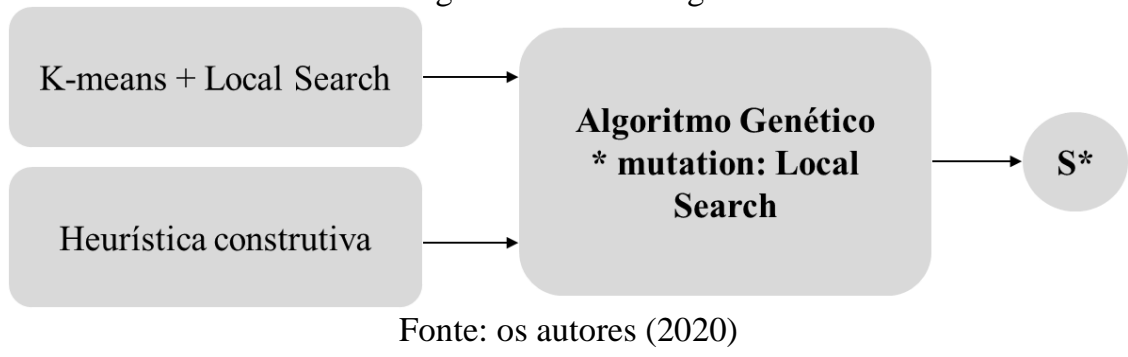

A população inicial é formada por dez indivíduos, o primeiro é obtido por meio do $k$-means melhorado pelo Local Search e a outros nove por uma heurística construtiva.

No k-means os pontos centrais dos clusters são escolhidos aleatoriamente. Após, é realizado o cálculo da distância entre todos os pontos e o ponto central (centroide) de cada cluster. Os pontos são designados para o cluster mais próximo até que todos tenham sido alocados. Os centroides são recalculados a partir da média de todos os elementos pertencentes ao cluster $\mathrm{k}$. O algoritmo continua a atribuição dos clusters e cálculo dos centroides até que não haja diferença entre a solução atual e a anterior. Meilă (2006) destaca que o k-means é um algoritmo que converge rapidamente para um ótimo local. A partir da solução inicial obtida pelo k-means é realizada uma busca em sua vizinhança com a aplicação do Local Search. A cada iteração a melhor solução é guardada. O critério de parada estabelecido é de 20 iterações sem melhoria. 
É utilizada uma heurística construtiva para obtenção de soluções iniciais variadas com o intuito aumentar a diversidade dos cruzamentos. Na heurística construtiva o ponto central de cada cluster é escolhido aleatoriamente e os outros pontos são designados para o cluster mais próximo. Diferente do k-means, o processo é feito apenas uma vez, sem recalcular os centroides.

Três indivíduos são selecionados aleatoriamente, os dois com menor distância são escolhidos para a realização do cruzamento (crossover), o ponto de troca é selecionado de forma aleatória. São obtidas duas novas soluções a partir do crossover das soluções selecionadas. As duas piores soluções da população anterior são removidas e as duas novas são adicionadas. Para cada um dos 10 indivíduos da população é sorteado um número aleatório entre 0 e 1, os indivíduos que obtiverem valor menor ou igual a 0,2 sofrerão uma mutação em sua estrutura através da aplicação do Local Search. O método continua até que sejam obtidas dez iterações sem melhoria.

Tabela 3 - Pseudocódigo

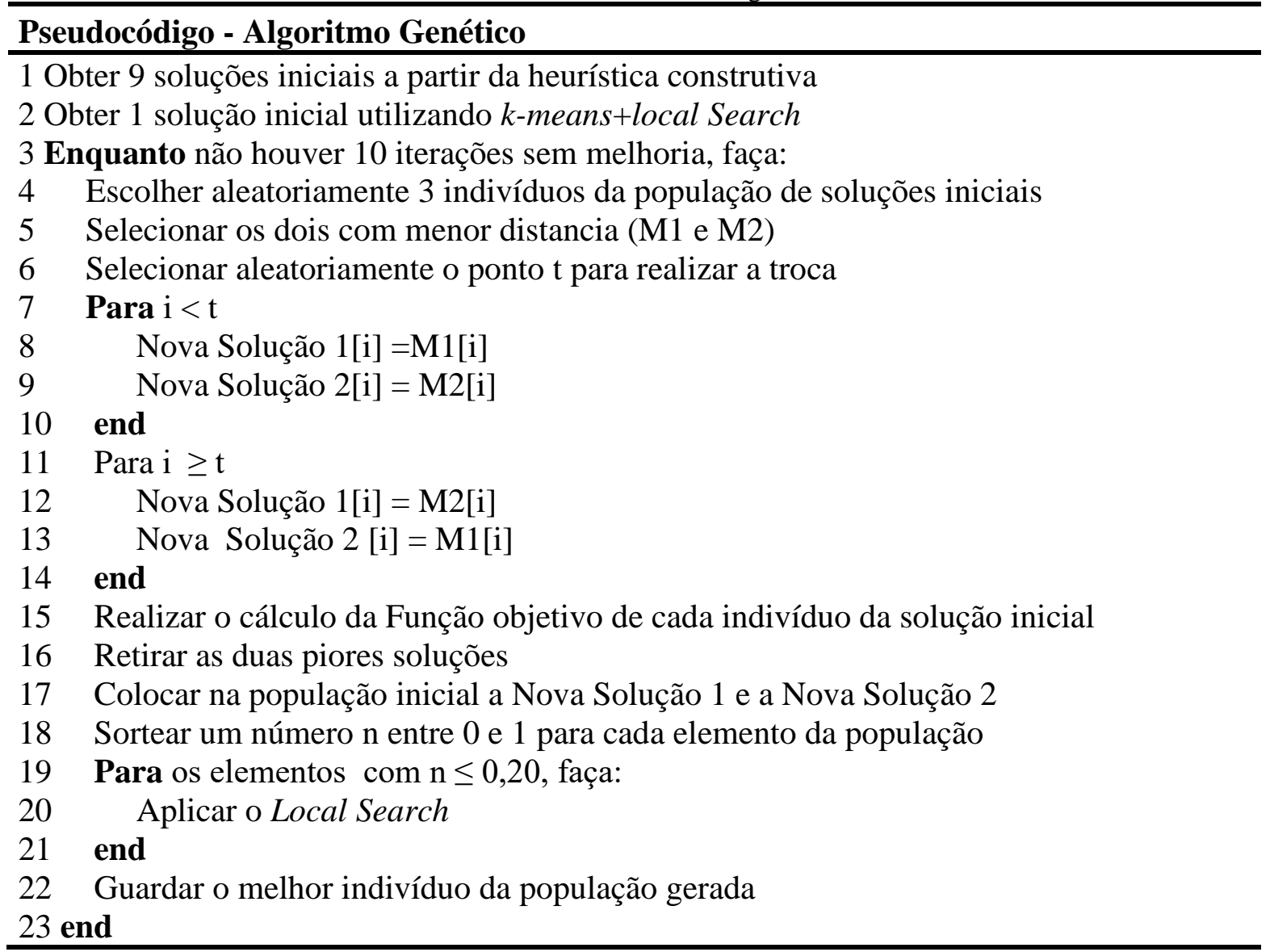




\section{Resultados computacionais}

Foram resolvidas 47 instâncias geradas aleatoriamente com 2,4,7 e 10 clusters e 20, 50 e 200 vértices. Foi utilizado o Visual Basic 2017 na linguagem de programação C\# em um computador Intel core i5-8250U, com $8 \mathrm{~Gb}$ de memória ram. O modelo exato foi resolvido com o software Gurobi 8.0 em suas configurações padrões.

Como critério de tempo computacional para a obtenção da solução foi determinado o tempo máximo de seis horas para cada instância.

Os resultados computacionais são apresentados na tabela 4. Como medida de comparação foi utilizado o tempo computacional para obter a solução e o GAP entre a metodologia proposta e o modelo exato que é determinado pela fórmula: $\mathrm{Sol}_{m}-\mathrm{Sol}_{\boldsymbol{g}} / \mathrm{Sol}_{m}$, onde $\mathrm{Sol}_{m}$ é a solução obtida com a metaheurística e $\operatorname{Sol}_{e}$ a solução do modelo exato. É possível observar que a metodologia proposta alcança o resultado ótimo em $86,21 \%$ das instâncias com até 50 pontos. Para as instâncias onde não foi possível obter a solução ótima com a metaheurística o maior GAP encontrado foi de 3,63\%. Para 50 pontos e 4 clusters o modelo exato aumenta consideravelmente o tempo de resolução, até que em instâncias com 200 pontos não é possível obter a resposta no tempo máximo de 6 horas. O AG resolve instâncias com 200 vértices em um tempo computacional baixo, não ultrapassando 7 minutos.

Tabela 3 - Resultados

\begin{tabular}{|c|c|c|c|c|c|c|c|}
\hline Instância & Pontos & Clusters & $\begin{array}{c}\text { FO } \\
\text { Metodologia } \\
\text { Proposta }\end{array}$ & Tempo(s) & $\begin{array}{c}\text { FO do } \\
\text { modelo } \\
\text { exato }\end{array}$ & Tempo(s) & GAP \\
\hline 1 & 20 & 2 & 8348,68 & 0,67 & 8348,68 & 0,35 & $0,00 \%$ \\
\hline 2 & 20 & 2 & 8009,9 & 0,57 & 8009,9 & 0,14 & $0,00 \%$ \\
\hline 3 & 20 & 2 & 8201,6 & 0,48 & 8201,6 & 0,05 & $0,00 \%$ \\
\hline 4 & 20 & 2 & 6891,74 & 0,48 & 6891,74 & 0,03 & $0,00 \%$ \\
\hline 5 & 20 & 2 & 8410,65 & 0,51 & 8410,65 & 0,07 & $0,00 \%$ \\
\hline 6 & 20 & 2 & 9365,53 & 0,5 & 9365,53 & 0,09 & $0,00 \%$ \\
\hline 7 & 20 & 2 & 7845,59 & 0,51 & 7845,59 & 0,07 & $0,00 \%$ \\
\hline 8 & 20 & 2 & 6390,47 & 0,49 & 6390,47 & 0,19 & $0,00 \%$ \\
\hline 9 & 20 & 2 & 9667,85 & 0,48 & 9667,85 & 0,17 & $0,00 \%$ \\
\hline 10 & 20 & 2 & 8991,27 & 0,45 & 8991,27 & 0,07 & $0,00 \%$ \\
\hline 11 & 20 & 2 & 12523,03 & 0,55 & 12523,02 & 0,25 & $0,00 \%$ \\
\hline 12 & 20 & 2 & 12014,85 & 0,55 & 12014,85 & 0,11 & $0,00 \%$ \\
\hline 13 & 20 & 2 & 10866,39 & 0,51 & 10866,39 & 4,76 & $0,00 \%$ \\
\hline 14 & 20 & 2 & 10337,61 & 0,49 & 10337,61 & 0,06 & $0,00 \%$ \\
\hline 15 & 20 & 2 & 12615,98 & 0,49 & 12615,98 & 0,06 & $0,00 \%$ \\
\hline 16 & 20 & 2 & 14048,29 & 0,51 & 14048,29 & 0,07 & $0,00 \%$ \\
\hline
\end{tabular}




\begin{tabular}{rrrrrrrr}
17 & 20 & 2 & 10896,24 & 0,52 & 10896,24 & 0,4 & $0,00 \%$ \\
18 & 20 & 2 & 9585,71 & 0,54 & 9585,71 & 0,06 & $0,00 \%$ \\
19 & 20 & 2 & 14293,55 & 0,5 & 14293,55 & 0,25 & $0,00 \%$ \\
20 & 20 & 2 & 13486,91 & 0,49 & 13486,91 & 0,35 & $0,00 \%$ \\
21 & 50 & 2 & 102967,1 & 8,4 & 102967,1 & 4,12 & $0,00 \%$ \\
22 & 50 & 4 & 27353,42 & 3,76 & 27175,58 & 8381,23 & $0,65 \%$ \\
23 & 50 & 4 & 32614,42 & 3,77 & 32359,9 & 19952,63 & $0,78 \%$ \\
24 & 50 & 4 & 27483,05 & 4,31 & 27483,05 & 2072,32 & $0,00 \%$ \\
25 & 50 & 4 & 29114,41 & 3,12 & 29114,41 & 179,19 & $0,00 \%$ \\
26 & 50 & 4 & 30562,3 & 4,31 & 30562,3 & 4836,12 & $0,00 \%$ \\
27 & 50 & 4 & 33863,75 & 3,55 & 32634,85 & 9443,27 & $3,63 \%$ \\
28 & 50 & 4 & 30824,74 & 3,61 & 30824,74 & 273,26 & $0,00 \%$ \\
29 & 50 & 4 & 29154,91 & 3,49 & 28923,1 & 1568,17 & $0,80 \%$ \\
30 & 200 & 4 & 593911,3 & 414,77 & - & - & - \\
31 & 200 & 4 & 780548,61 & 403,37 & - & - & - \\
32 & 200 & 4 & 865205,03 & 936 & - & - & - \\
33 & 200 & 4 & 926090,71 & 385,95 & - & - & - \\
34 & 200 & 4 & 940394,92 & 405,56 & - & - & - \\
35 & 200 & 4 & 657299,91 & 409,97 & - & - & - \\
36 & 200 & 4 & 724547,7 & 469,26 & - & - & - \\
37 & 200 & 7 & 245336,09 & 318,82 & - & - & - \\
38 & 200 & 10 & 266096,31 & 193,457 & - & - & - \\
39 & 200 & 10 & 211931,55 & 239,47 & - & - & - \\
40 & 200 & 10 & 208012,1 & 165,43 & - & - & - \\
41 & 200 & 10 & 238713,72 & 225,12 & - & - & - \\
42 & 200 & 10 & 194576,94 & 217,39 & - & - & - \\
43 & 200 & 10 & 188248,07 & 174,42 & - & - & - \\
44 & 200 & 10 & 238591,99 & 178,04 & - & - & - \\
45 & 200 & 10 & 202627,39 & 191,94 & - & - & - \\
46 & 200 & 10 & 225438,37 & 183,7 & - & - & - \\
47 & 200 & 10 & 173109,61 & 251,66 & - & - & - \\
\hline & & & fonte: Os autores (2020) & & &
\end{tabular}

\section{Considerações finais}

O objetivo do presente estudo foi propor uma metodologia utilizando metaheurísticas para a resolução do problema de mínima distância entre vértices pertencentes a um cluster para a resolução de problemas onde não é possível obter a solução exata em um tempo computacional baixo.

A metodologia proposta consiste na utilização do Algoritmo Genético a partir de soluções iniciais obtidas por uma heurística construtiva e pelo k-means melhorado pelo Local Search. 
O Algoritmo Genético é utilizado pois o k-means converge rapidamente para um ótimo local enquanto o AG realiza uma busca global.

Para testar a eficiência da metodologia o modelo exato proposto por Nascimento, Todelo e Carvalho (2010) foi resolvido de forma exata e os resultados da função objetivo e tempo computacional foram comparados com o AG.

Como resultado da pesquisa obteve-se que a metodologia proposta é eficiente pois obtém a solução ótima em pequenas instâncias e nas maiores consegue uma solução em tempo computacional baixo enquanto o modelo exato não obtém resposta.

\section{REFERÊNCIAS}

BARBOZA, A. O. Simulação e técnicas da computação evolucionária aplicadas a problemas de programação linear inteira mista. 2010.

CAO, L. et al. Automatic feature group combination selection method based on GA for the functional regions clustering in DBS. Computer Methods and Programs in Biomedicine, v. $183,2020$.

CASTRO, R. E. DE. Otimização de estruturas com multi-objetivos via algoritmos genéticos. PhD, p. 206, 2001.

CHAO, C.; ZHIHUI, T.; BAOZHEN, Y. Optimization of two-stage location-routinginventory problem with time-windows in food distribution network. Annals of Operations Research, v. 273, n. 1-2, p. 111-134, 2019.

DEFRYN, C.; SÖRENSEN, K.; CORNELISSENS, T. The selective vehicle routing problem in a collaborative environment. European Journal of Operational Research, 2016.

FUENTES, M.; CADARSO, L.; MARÍN, Á. A hybrid model for crew scheduling in rail rapid transit networks. Transportation Research Part B: Methodological, 2019.

GATICA, G. et al. Efficient heuristic algorithms for location of charging stations in electric vehicle routing problems. Studies in Informatics and Control, v. 27, n. 1, p. 73-82, 2018.

GEREMEW, W. et al. A DC programming approach for solving multicast network design problems via the Nesterov smoothing technique. Journal of Global Optimization, v. 72, n. 4, p. 705-729, 2018.

JAIN, A. K. Data clustering: 50 years beyond K-means. Pattern Recognition Letters, v. 31, n. 8, p. 651-666, 2010.

LOPES, H. S.; RODRIGUES, L. C. DE A.; ARNS, M. T. S. Meta-Heurísticas em Pesquisa Operacional. Editora Omnipax. 2013. 
MEILǍ, M. The uniqueness of a good optimum for K-means. ACM International Conference Proceeding Series, v. 148, p. 625-632, 2006.

NASCIMENTO, M. C. V.; TOLEDO, F. M. B.; DE CARVALHO, A. C. P. L. F. Investigation of a new GRASP-based clustering algorithm applied to biological data. Computers and Operations Research, v. 37, n. 8, p. 1381-1388, 2010.

TALBI, M. E. Metaheuristics : from Design to Implementation Single solution-based metaheuristics. John Wiley \& Sons. 2009.

TIWARI, A.; CHANG, P. C. A block recombination approach to solve green vehicle routing problem. International Journal of Production Economics, 2015.

WANG, Y. et al. Collaborative multi-depot logistics network design with time window assignment. Expert Systems with Applications, v. 140, 2020.

WU, Z.; ZHAO, C.; LIU, B. Polygonal Approximation based on Coarse-grained Parallel Genetic Algorithm. Journal of Visual Communication and Image Representation, p. $102717,2019$. 